\title{
Immunologie - die Basis der Allergologie
}

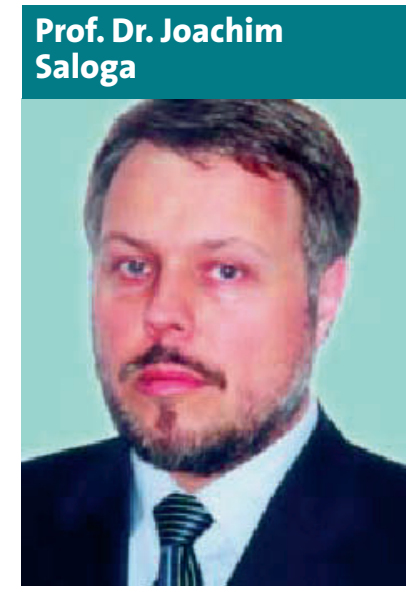

Universitäts-Hautklinik Mainz, Sprecher der Sektion Immunologie der DGAI

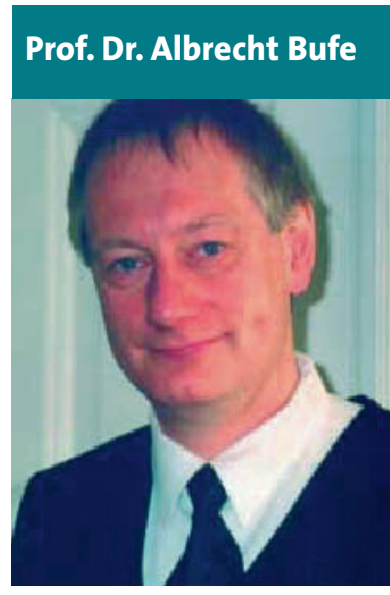

Experimentelle Pneumologie, Universität Bochum, stellvertretender Sprecher der Sektion Immunologie der DGAI Immunologie der Deutschen Gesellschaft für Allergologie und klinische Immunologie (DGAI) erarbeitet wurde, stellt den Versuch dar, in äußerst knapper Form wichtige immunologische Grundlagen der Allergologie auf aktuellem Stand darzustellen. Besondere Betonung finden Aspekte, die für die Praxis der Allergologie relevant sind, und neuere

\section{Kontakt}

\section{Sektion Immunologie der DGAI}

Prof. Dr. med. Joachim Saloga

Universitäts-Hautklinik Mainz

Langenbeckstr. 1

55131 Mainz

Fax: (o 61 31) 17473751

E-Mail: saloga@hautklinik.klinik. uni-mainz.de.

Erkenntnisse, die sich auf dem sich rasch entwickelnden Gebiet der Immunologie zahlreich eingestellt haben.

In diesem Umfang ist es natürlich nicht möglich die Immunologie umfassend darzustellen, so dass diesbezüglich auf die aktuellen Lehrbücher der Immunologie verwiesen werden muss. Dennoch wird hier ein weiter Bogen

geschlagen von den Allergenmolekülen als Antigenen und der Immungenetik als wichtigem anlagebedingten Steuerungsfaktor über die wichtigsten Immunzellen - wie antigenpräsentierende Zellen, T- und B-Lymphozyten sowie eosinophile und basophile Granulozyten und Mastzellen - bis hin zu den molekularen Prozessen der Zelladhäsion und -migration sowie modulierenden Einflüssen seitens des Nervensystems oder durch Infekte. Weiterhin wird das anatomisch und funktionell besonders gestaltete Immunsystem der allergologisch bedeutsamen Organe Atemwege/Lunge, Haut und Gastro-

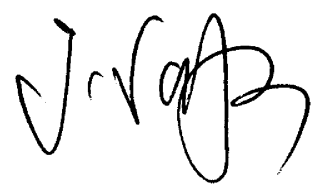

Prof. Dr. Joachim Saloga intestinaltrakt im Hinblick auf die wichtigsten allergischen Krankheiten dieser Organe, nämlich Asthma bronchiale, Ekzeme und allergische Erkrankungen des Verdauungstraktes, dargestellt. Schließlich werden die allergologische Labordiagnostik und immunologisch basierte Therapieverfahren vor dem dazu gehörenden immunologischen Hintergrund erläutert - manche allerdings erst in der kommenden Ausgabe 3 des Allergo Journal (Erscheinungstermin 29. April 2003), denn alle Beiträge waren beim besten Willen nicht in einer einzigen Ausgabe unterzubringen.

Die Sektion Immunologie hofft, mit dem Themenheft Immunologie einen Beitrag zu einem besseren Verständnis immunologischer Mechanismen bei allergischen Krankheiten sowie deren Diagnostik und Therapie geleistet zu haben. Falls durch dieses Heft Mitglieder der DGAI angeregt worden sind, sich an der Arbeit der Sektion Immunologie aktiv zu beteiligen, sind sie herzlich eingeladen sich der Sektion Immunologie anzuschließen.

Mit kollegialen Grüßen, 